\title{
Implementación de herramientas de calidad en una empresa textil del Bajío
}

\section{Implementation of quality tools in a textile company in Bajío}

\author{
CALDERÓN-GONZÁLEZ, Giuliana $\dagger^{*}$, ESPINOSA-RODRIGUEZ, Marcela y OLVERA- \\ MONTOYA, Ana Luisa
}

Instituto Tecnológico Superior de Salvatierra, Tecnológico Nacional de México

ID $1^{\text {er }}$ Autor: Giuliana, Calderón-González / ORC ID: 0000-0003-2670-6004, Researcher ID Thomson: X-3088-2018, CVU CONACYT ID: 953348

ID $1^{\text {er }}$ Coautor: Marcela, Espinosa-Rodríguez / ORC ID: 0000-0001-9874-3432, Researcher ID Thomson: X- 3049-2018, CVU CONACYT ID: 523175

ID $2^{\text {do }}$ Coautor: Ana Luisa, Olvera-Montoya / ORC ID: 0000-0001-5922-674X, Researcher ID Thomson: X-2907-2018, CVU CONACYT ID: 953288

DOI: $10.35429 /$ JTIP.2019.7.3.6.11

Recibido 03 de Abril, 2019; Aceptado 09 Junio, 2019

\section{Resumen}

El objetivo de la investigación es implementar herramientas de calidad en una empresa textil del bajío; dicha investigación es mixta, ya que se obtienen datos a través de la observación, entrevistas y se realizan gráficos para analizar la información. Se inició conociendo el proceso de producción de tela, las máquinas involucradas en el proceso, las etapas que lo conforman y las variables a controlar. Posteriormente se registró las variables del proceso en el software Minitab, para predecir a través de los gráficos de control la variación que presenta el proceso. Con respecto a los resultados obtenidos, se determinó el tipo de herramientas implementadas. Las herramientas de calidad aplicadas fueron las cartas de control X - R y X $\mathrm{S}$ para conocer la capacidad del proceso. Diagrama de Pareto para conocer los defectos de calidad frecuentes. Análisis del modo y efecto de fallas (AMEF), para visualizar los defectos en cada etapa de producción, las causas que las originan e identificar qué tipo de control debía ser aplicado. Se contribuyó a identificar puntos críticos y mejorar la calidad en los procesos de producción, que permiten ofrecer a los clientes productos de alta calidad y enfrentar la competencia a nivel local, nacional e internacional.

Calidad, defectos y AMEFF

\begin{abstract}
The objective of the research is to implement quality tools in a low-year textile company; such research is mixed, as data are obtained through observation, interviews and graphs are made to analyze the information. He began knowing the process of fabric production, the machines involved in the process, the stages that make up it and the variables to control. Subsequently, the process variables were recorded in the Minitab software, to predict through the control charts the variation presented by the process. With respect to the results obtained, the type of tools implemented was determined. The quality tools applied were the control charts $\mathrm{X}-\mathrm{R}$ and $\mathrm{X}-\mathrm{S}$ to know the capacity of the process. Pareto diagram for frequent quality defects. Analysis of the mode and failure effect (AMEF), to visualize the defects at each stage of production, the causes that cause them and identify what type of control should be applied. It helped to identify critical points and improve the quality in production processes, which allow customers to offer high quality products and face competition at the local, national and international levels.
\end{abstract}

Quality, defects and AMEFF

Citación: CALDERÓN-GONZÁLEZ, Giuliana, ESPINOSA-RODRIGUEZ, Marcela y OLVERA-MONTOYA, Ana Luisa. Implementación de herramientas de calidad en una empresa textil del Bajío. Revista de Tecnologías en Procesos Industriales. 2019. 3-7: 6-11

\footnotetext{
* Correspondencia del Autor (gicalderon@itess.edu.mx)

$\uparrow$ Investigador contribuyendo como primer autor.
} 


\section{Introducción}

Uno de los puntos claves para asegurar el éxito y el correcto funcionamiento de la empresa es asegurarse de realizar un control de calidad de todos los procesos que conlleva la actividad empresarial, con el objetivo de obtener una mejora continua de la organización que nos permita sacar la mayor rentabilidad de nuestro negocio, a la vez que se satisfacen las necesidades de los clientes y estos posee el mayor grado de satisfacción (Caurin, 2018).

La gran mayoría de empresas mexicanas, debido a recurrentes crisis provocadas por una inestabilidad económica mundial y por situaciones políticas que generan desconfianza en los mercados mundiales y nacionales en nuestro entorno, así como el proteccionismo establecido por las autoridades, no ha permitido que éstas se preparen adecuadamente para competir de una manera igualitaria en lo que se refiere al concepto de calidad, con las empresas transnacionales e importaciones desleales que nos afectan día con día (Grupo ALBE, 2018).

La empresa textil del Bajío dedicada a la elaboración de diferentes tipos de productos textiles como lo son hilos y telas de diferentes tipos de acabado tiene como objetivo mantener a sus clientes satisfechos con el producto que se les ofrece y hacer que logre satisfacer las necesidades para las cuales fue fabricado.

Esto es posible solo si se tiene un proceso controlado y una adecuada inspección dentro de cada una de las etapas que lo componen. Aunque como bien es sabido, dentro de todo proceso se encuentra presente la variación; sin embargo, se tienen que diseñar estrategias que ayuden a minimizarla para evitar que el proceso salga de control y como consecuencia afecte la calidad del producto final.

Para lograr el objetivo de la empresa textil, se realizó este proyecto que permitió identificar el estado de calidad de la tela, las variables criticas del proceso y la implementación de herramientas de calidad para mejorar continuamente y por ende satisfacer las necesidades de los clientes internos y/o externos, así como potenciar la competitividad en el mercado local, nacional e internacional.

\section{Problema}

La empresa Textil del Bajío en los últimos meses ha detectado que algunos rollos de la tela procesada no cumplen con los estándares de calidad establecidos, ya sea por el cliente o por la misma empresa; esto como consecuencia de una serie de variables que afectan el proceso, provocando que laboratorio no libere la tela y de acuerdo a la gravedad pueda o no ser reprocesada. La tela procesada debe ser evaluada al final del mismo, esto con el fin de saber si cumple con los estándares de calidad y el encargado de hacerlo es laboratorio físico.

De acuerdo a los registros que se tienen, aproximadamente el $20 \%$ de los rollos no cumplen con los estándares de calidad ya sea por flama, quiebre, manchas o cualquier otro defecto.

Es por ello que surgió la necesidad de que dicho proceso se encuentre bajo una constante inspección en cada una de las áreas que lo conforman, las cuales corresponden a preparación, teñido y acabado de las telas, para identificar las variables que se tienen que medir y controlar; así como las medidas correspondientes que puedan permitir mejorarlo.

\section{Objetivos}

\section{Objetivo general}

Implementar herramientas de calidad en una empresa textil del bajío.

\section{Objetivos específicos}

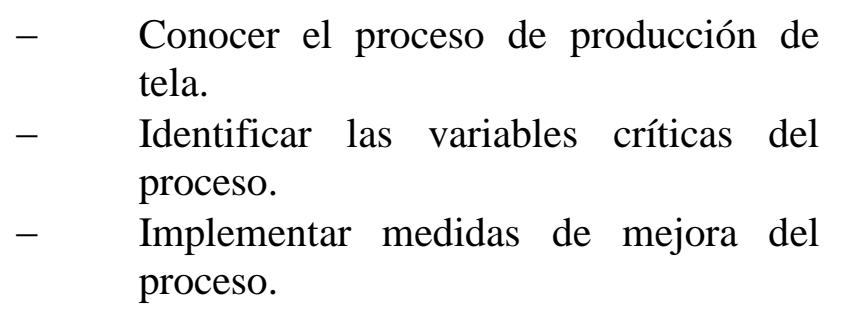

\section{Marco Teórico \\ El marco teórico utilizado se menciona a continuación:}




\section{- $\quad$ Calidad}

La calidad supone que el producto o servicio deberá cumplir con las funciones y especificaciones para las que ha sido diseñado y que deberán ajustarse a las expresadas por los consumidores o clientes (Cuatrecasas, 2010).

\section{- $\quad$ Diagrama de Pareto.}

Es un gráfico especial de barras cuyo campo de análisis o aplicación son los datos categóricos cuyo objetivo es ayudar a localizar el o los problemas vitales, así como sus causas más importantes (Gutiérrez, 2010).

\section{- $\quad$ Graficas de control.}

El objetivo básico de una carta de control es observar y analizar el comportamiento de un proceso a través del tiempo. Así es posible distinguir entre variaciones por causas comunes y especiales (atribuibles), lo que ayudara a caracterizar el funcionamiento del proceso y decidir las mejores acciones de control y de mejora (Gutiérrez, 2013).

- Análisis de modos y efectos de falla (AMEF).

El AMEF es una metodología que permite analizar la calidad, seguridad y/o fiabilidad del funcionamiento de un sistema, tratando de identificar las fallas potenciales que presenta su diseño y por lo tanto tratando de prevenir problemas futuros de calidad. Se aplica por medio del estudio sistemático de las fallas y sus causas partiendo de sus efectos (Delgado, 2011).

\section{Metodología a desarrollar}

\section{Tipo de investigación}

En un enfoque mixto el investigador utiliza las técnicas de cada uno por separado, se hacen entrevistas, se realizan encuestas para saber las opiniones de cada cual sobre el tema en cuestión, se trazan lineamientos sobre las políticas a seguir según las personas que intervengan, etc. Se obtienen rangos de valores de las respuestas, se observan las tendencias obtenidas, las frecuencias, se hacen histogramas, se formulan hipótesis que se corroboran posteriormente (Cortés, 2004).
Por lo mencionado anteriormente, se realizó la observación del proceso, se recolectaron datos y se cuestionó a las personas involucradas en el proceso para poder determinar las causas que afectan la calidad de la tela; así como se elaboraron graficas que permitieron observar el comportamiento del proceso para poder utilizar herramientas que permitieran mejorar la calidad del producto terminado.

\section{Métodos Teóricos}

\section{Conocimiento del proceso de producción de tela}

Se acudió a la empresa con el fin de conocer las etapas que conforman el proceso de producción de la tela. Dicho proceso está dividido en tres áreas: preparación, acabado y acabado final.

En el área de preparación, la tela cruda pasa por cuatro diferentes etapas del proceso. Posteriormente, el área de acabados, está conformada por tres etapas y en la etapa final se realizan las actividades necesarias para cumplir con los requerimientos del cliente.

En cada una de las diferentes etapas se observó la serie de pasos a realizar, así como las diferentes variables involucradas.

Se observaron los datos históricos de los reportes semanales, los cuales contienen información de las variables medidas en cada uno de los productos procesados, así como algunos gráficos de control.

Después se realizó una entrevista oral a los operarios del proceso de producción, con el fin de saber su opinión respecto a las variables que se medían, las condiciones que influían en el proceso y los tipos de defectos que presentaba la tela en cada una de las etapas.

\section{Identificación de las variables críticas del proceso}

Por medio de la observación, los datos históricos y las entrevistas realizadas se determinó las variables que se tenían que monitorear. Se comenzó la recolección de datos en las tres diferentes etapas del proceso, revisando la calidad de la tela antes y después de procesarse; con el objetivo de identificar los defectos y saber el origen o causa de los mismos. 
Se procedió a analizar las variables que eran monitoreadas e inspeccionar los rollos que estaban fuera de los límites establecidos. Realizando pruebas de calidad, tomando una muestra de tela de aproximadamente de $30 \mathrm{~cm}$ de ancho y llevada a laboratorio para que se le hicieran las pruebas correspondientes.

Posteriormente los datos recolectados fueron capturados en el softwarew Minitab V.17, para poder predecir a través de los gráficos de control la variación que se presenta en la primera etapa del proceso.

\section{Implementación de medidas de mejora del proceso}

Los datos obtenidos permitieron tomar la decisión de aplicar cartas de control X - R y X$\mathrm{S}$ para conocer la capacidad del proceso y en base a ello interpretar el comportamiento del proceso.

El diagrama de Pareto para identificar cuál de los defectos de calidad se debe atacar, así como las causas que lo originan.

Fue elaborado un AMEFF con el objetivo de visualizar los defectos de la tela en cada una de las etapas del proceso de producción, conocer las causas del porque se estaban originando; así como identificar qué tipos de controles debían ser aplicados para lograr reducir dichos defectos.

\section{Resultados}

1. Se realizó un diagrama de bloques del proceso, tal como se muestra en el Anexo 1.

La información de las entrevistas mostro que la tela presenta diversos defectos en cada una de las etapas que conforman dicho proceso, los cuales son: manchas de borra, aceite, grasa, tierra, etc., grumos, roses, arrugas, hilo faltante, hilo contaminado, piojo, rigidez y quiebres.

Del análisis realizado se concluye que el problema que afecta la calidad del producto es quiebres, como se observa en la figura 1.

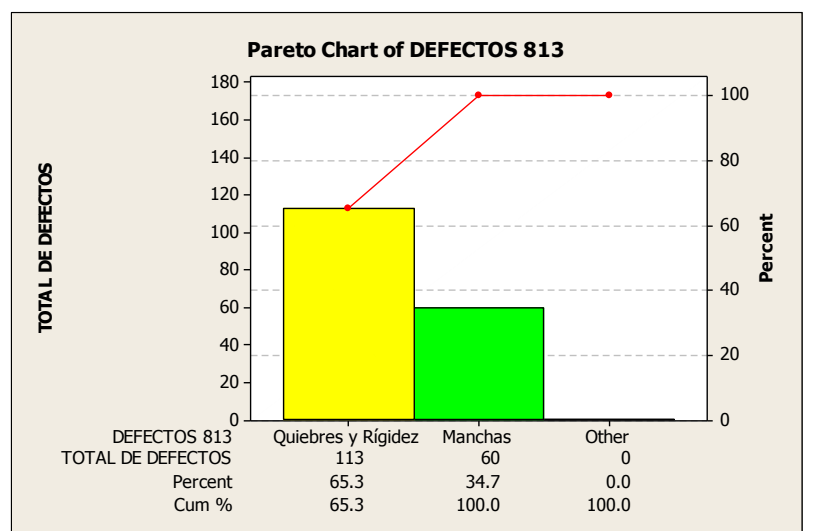

Figura 1 Grafica de Pareto de defectos de tela

2. Las variables monitoreadas son:

Condición "A" que obtiene la tela en la primera etapa, velocidad, temperatura, presiones de foulares, porcentaje de arrastre. La condición "A", se refiere a la variable más crítica que se mide en el proceso, es llamada así; por motivos de confidencialidad.

Se determinó que existen otras variables que afectan al proceso y no son monitoreadas: capacidad de absorción, ancho de la tela, porcentaje de concentración de producto.

La norma ASTM D-6413, menciona que la especificación de calidad es: longitud carbonizada $\leq 10 \mathrm{~cm}$. La figura 2 , muestra que los niveles de condición "A" (normal, alta y baja) en que se procesa la tela no afecta la calidad del producto.

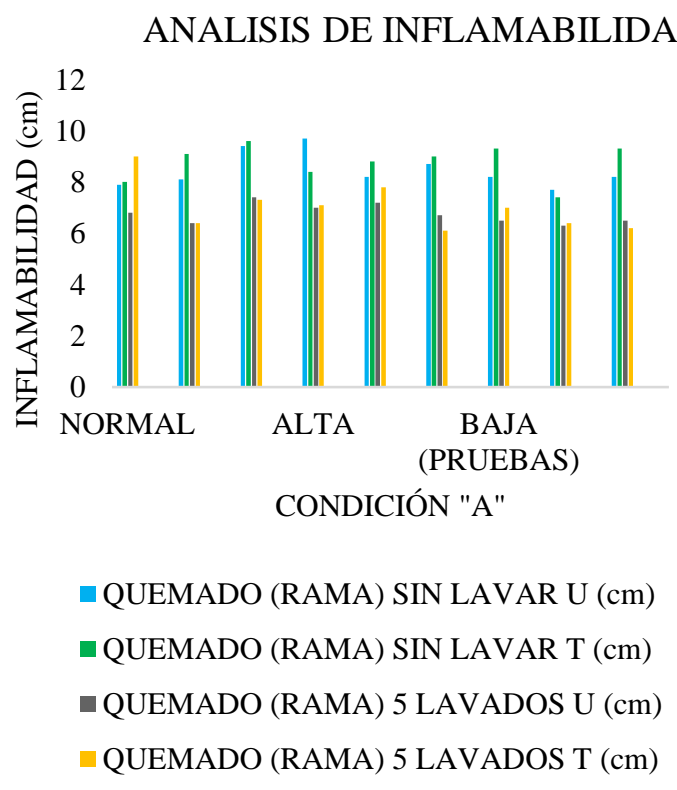

Figura 2 Grafica de inflamabilidad 
Después de realizar diferentes pruebas se concluyó que los límites de la condición "A" deben ser: $9-10 \%$.

Los resultados de la variable de capacidad de absorción mostraron que no cumplen con los estándares establecidos: mínimo de tres $\mathrm{cm}$ de absorción.

Con respecto a las pruebas de concentración de producto realizadas por el laboratorio químico, el resultado fue: $4.3 \%$ de concentración; valor superior al límite máximo establecido: $3.6 \%$.

3. Con las pruebas realizadas, se determina que las variables a controlar son:

Condición "A" (9 - $10 \%)$, capacidad de absorción, concentración de producto y ancho. Se realizó un cambio en los límites de la condición "A" y se elimina el control de las variables monitoreadas: presiones de Foulard, pickup, temperaturas y velocidad.

Con límite superior a $10 \%$, se presentan 20 puntos fuera de control. En la figura 3, se observa que aún hay rollos de tela fuera de control (cinco) con los nuevos límites establecidos de la condición "A"; por ello se deben buscar las causas que los originan.

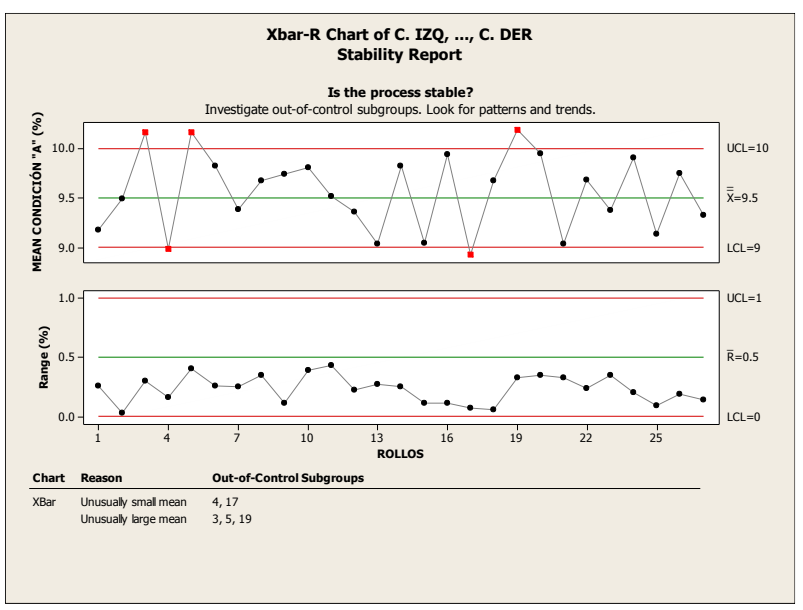

Figura 3 Grafica de control de condición "A" de rollos de tela

Se procede a realizar un AMEF de proceso (observar el Anexo 2) de la tela, el cual incluye los posibles defectos que pueden presentarse en las etapas que conforman el proceso, las causas y los controles de acción para corregir dicha problemática.
Los quiebres y la rigidez son los defectos más críticos con valores de RPN (número prioritario de riesgo) de 384 y 224, respectivamente; por ende, afectan la calidad de la tela y la venta de esta es de segunda calidad a un costo más bajo.

Por otro lado, las manchas ocasionadas por borra, fallas mecánicas o un proceso inadecuado obtuvieron valores RPN mayores a 100. Por lo tanto, son puntos críticos del proceso y se deben implementar estrategias para mejorar la calidad del producto terminado.

\section{Conclusiones}

Con los resultados obtenidos se puede concluir que el problema que afecta la calidad es quiebres.

Los límites de la variable condición "A" deben ser: $9-10 \%$.

Las variables que se controlaban no son significativas para controlar la calidad, por lo tanto, las variables que se deben controlar son: condición "A", capacidad de absorción, concentración de producto y ancho.

En el AMEF se identifica los quiebres y la rigidez con RPN de 384 y 224 y las manchas con RPN mayores a 100 , por lo cual se establecieron acciones preventivas y correctivas para poder mejorar la calidad de la tela.

\section{Agradecimiento}

Al Instituto Tecnológico Superior de Salvatierra. A la empresa textil del Bajío y a la estudiante Verónica Martínez Serrato por su apoyo en la realización de esta investigación

\section{Referencias}

Caurin, J. (2018). Control de Calidad en las Empresas. Emprende Pyme. Net. Recuperado de: https://www.emprendepyme.net/control-decalidad-en-las-empresas.

Cortés Cortés Manuel E., I. L. (2004). Generalidades sobre Metodología de la Investigación. México: Universidad Autonoma del Carmen.

Cuatrecasas, L. (2010). Gestión Integral de la Calidad. Barcelona: Profit Editorial. 
Delgado, H. C. (2011). Desarrollo de una cultura de calidad. México, DF: Mc Graw Hill.

Gutiérrez Pulido Humberto, d. 1. (2009). Control estadístico de calidad y seis sigma. México, D.F: Mc Graw Hill.

Gutiérrez Pulido Humberto, d. 1. (2013). Control Estadístico de la calidad y Seis Sigma. México, DF: Mc Graw Hill Education.

Grupo ALBE (2018). Competidores sustitutos. La calidad para las empresas. Recuperado de: https://www.grupoalbe.com/competidoressustitutos-la-calidad-para-las-empresas/

\section{Anexos}

Anexo 1 Diagrama de bloque del proceso

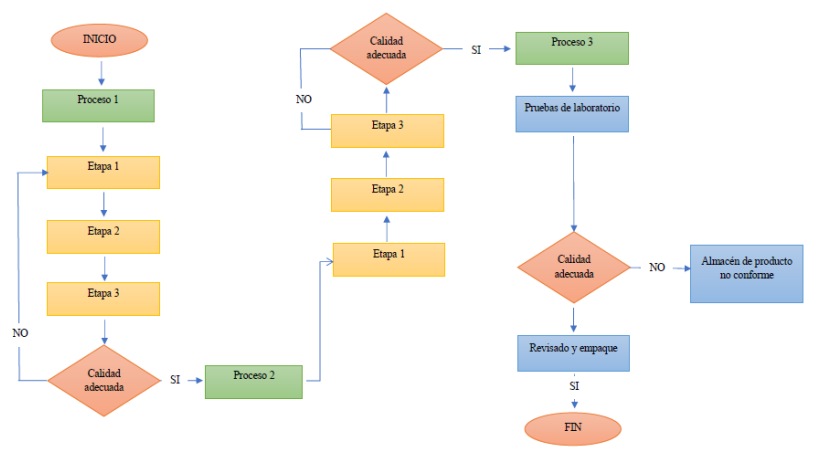

Anexo 2 AMEF de proceso de tela

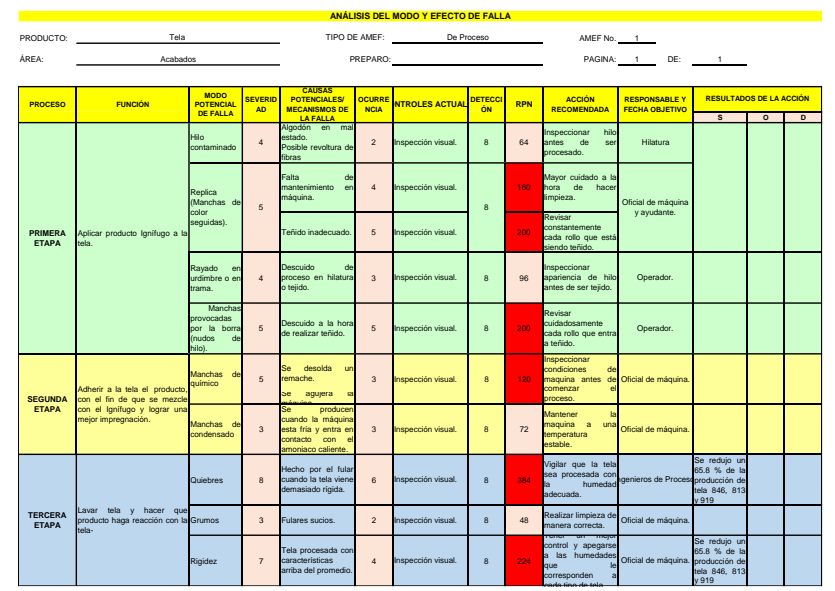

